\title{
Intraocular Endoscopy Resolved Tube Occlusion of an Ahmed Glaucoma Valve
}

\author{
Rumi Kawashima $^{a}$ Keita Babab Kenji Matsushita $^{a}$ Takeshi Soma ${ }^{a}$ \\ Masako Kurashige ${ }^{c}$ Daisuke Umeda ${ }^{c}$ Makoto Nakamura ${ }^{d}$ Eiichi Morii ${ }^{c}$ \\ Kohji Nishidaa, e \\ aDepartment of Ophthalmology, Osaka University Graduate School of Medicine, Suita, \\ Japan; 'bepartment of Ophthalmology, Nippon Life Hospital, Suita, Japan; 'Department \\ of Pathology, Osaka University Graduate School of Medicine, Suita, Japan; dDepartment of \\ Surgery, Division of Ophthalmology, Kobe University Graduate School of Medicine, Kobe, \\ Japan; 'Integrated Frontier Research for Medical Science Division, Institute for Open and \\ Transdisciplinary Research Initiatives, Osaka University, Suita, Japan
}

\section{Keywords}

Endoscopy $\cdot$ Ahmed glaucoma valve $\cdot$ Atopic dermatitis

\begin{abstract}
We report a case in which intraocular endoscopy clarified the cause of Ahmed glaucoma valve (AGV) failure with a cloudy cornea. A 42-year-old patient with glaucoma underwent AGV implant surgery to treat secondary glaucoma due to chronic iridocyclitis in his left eye. After AGV, he developed bullous keratopathy (BK) in that eye. After Descemet stripping automated endothelial keratoplasty (DSAEK) was performed to treat BK, the intraocular pressure (IOP) increased and early failure of the DSAEK resulted again in a cloudy cornea. We could not precisely detect any cause of AGV failure with ordinary imaging instrumentation. An intraocular endoscope was used to determine that cause, and we found that the fibrous tissue occluded the tube of the AGV. The IOP decreased soon after the tissue was removed. We conclude that intraocular endoscopy was useful for diagnosing AGV failure with BK.
\end{abstract}

\section{Karger $\stackrel{2}{\circ}$}




\section{Introduction}

Glaucoma is a leading cause of blindness, and reducing the intraocular pressure (IOP) is the only evidence-based treatment. Among glaucoma surgeries, trabeculectomy, the most common filtration surgery, carries the risk of severe postoperative complications [1]. In addition, bleb failure increases over time. The glaucoma drainage device (GDD) has played a significant role in the treatment of refractory glaucoma compared to any glaucoma surgeries. Some studies have reported that the GDD had a higher or similar success rate and fewer complications $[2,3]$. For these reasons, the use of GDD has increased dramatically in the USA and UK $[4,5]$.

Some unique complications are associated with the GDD, such as tube occlusion, tube erosion, tube-cornea touch, hypotony, bleb encapsulation, and diplopia [1, 6]. Among them, tube occlusion causes abnormally high IOP in either the early or late postoperative period. In the former, blood clots, iris, vitreous, or a lens-related substance proximally can obstruct the tube [7]; in the latter, the Ahmed glaucoma valve (AGV) (New World Medical, Inc., Rancho Cucamonga, CA, USA) causes distal tube occlusion by valve membrane adhesion [8] or fibrovascular ingrowth between the valve plate and cover [9]. However, late-onset proximal occlusion in the AGV remains poorly understood. We report a case in which intraocular endoscopy successfully visualized a rare cause of AGV tube occlusion in the late postoperative period and was helpful in the treatment.

\section{Case Presentation}

A 42-year-old man with atopic dermatitis underwent AGV implant surgery with tube insertion through the sulcus at another hospital to treat secondary glaucoma due to chronic iridocyclitis in his left eye. His right eye was blind because of a retinal detachment and secondary glaucoma. Six months after the implant surgery in his left eye, bullous keratopathy developed, and he was referred to our hospital (Fig. 1a). We performed Descemet stripping automated endothelial keratoplasty in the affected eye. The next day, the IOP increased to over $30 \mathrm{~mm} \mathrm{Hg}$. The patient had severe corneal cloudiness, which prevented visualization of ocular interior by slit-lamp microscopy. Using anterior-segment optical coherence tomography (AS-OCT) (CASIA2, Tomey, Nagoya, Japan), we confirmed that the AGV tube was in the superior temporal quadrant and filled with aqueous humor, and no filtering capsule blocked the tube (Fig. 1b). Although we observed a high-intensity area in the tube lumen under the scleral patch, we could not precisely detect the proximal tube occlusion around the tube tip through the ciliary sulcus because of poor mydriasis and backscatter from the posterior iris pigment (Fig. 1C).

We first performed a bleb needle revision using our method [10] to separate the fibrotic tissue around the valve cover from the AGV plate, but the IOP did not decrease. We then used intraocular endoscopy to detect the disrupted outflow from the tube into the AGV. No fibrovascular ingrowth was seen between the valve plate and valve cover, and no intra-valve hemorrhage was seen (Fig. 2a). When we observed the proximal drainage tube through the intraocular endoscope, we saw that the tube was occluded by the tissue (Fig. 2b); we used a lens capsule forceps to remove the tissue from the tube (Fig. 2c; online suppl. Digital Content 1; for all online suppl. material, see www.karger.com/doi/10.1159/000518286). We used hematoxylin and eosin staining to examine the surgically removed tissue embedded in paraffin, but nothing in the bright field microscopy images was stained, and the sample only showed a mass composed of small fragmented fibrous structures (Fig. 2d).

The IOP decreased to about $10 \mathrm{~mm} \mathrm{Hg}$ with antiglaucoma eye drops and remained stable for 1 year postoperatively. The patient underwent penetrating keratoplasty for recurrent 

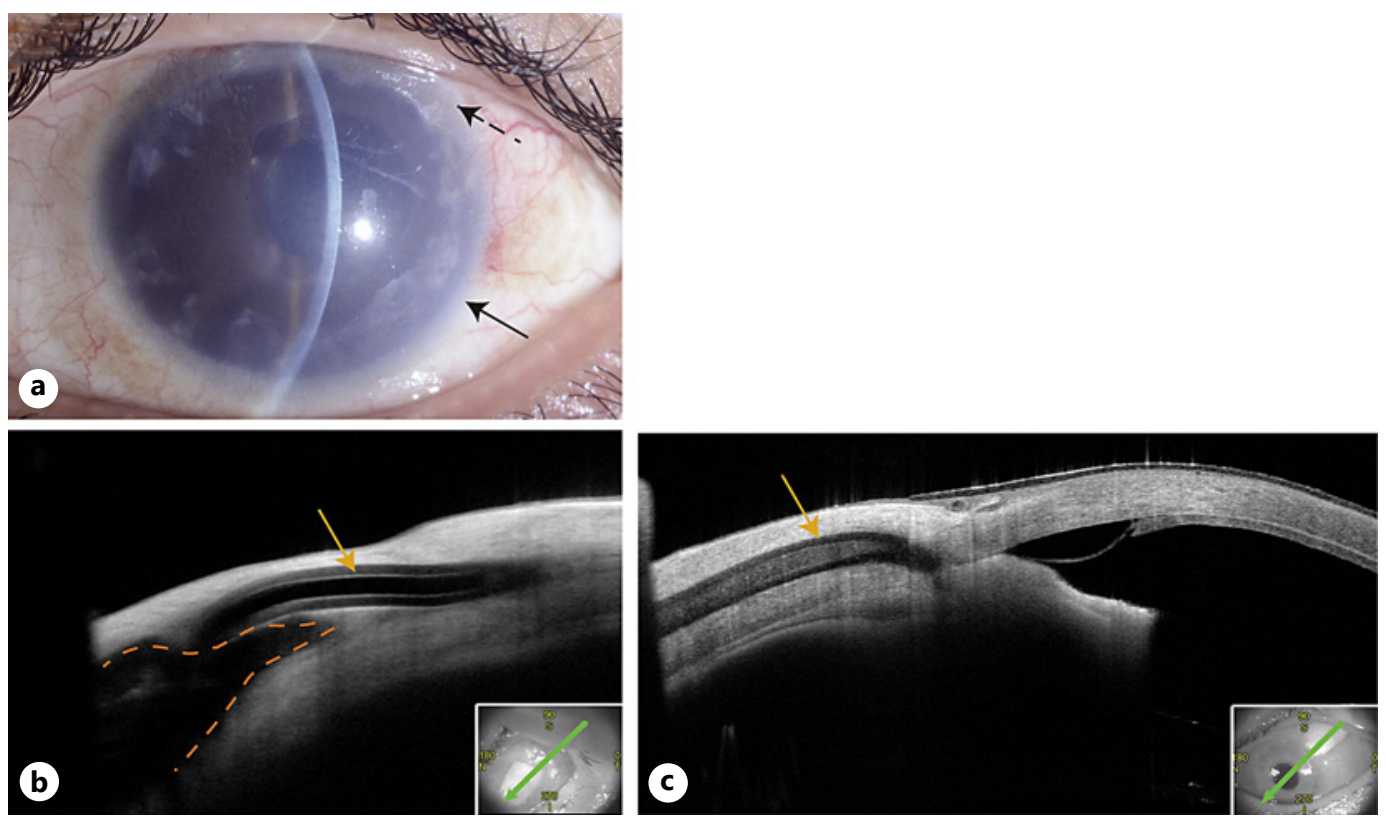

Fig. 1. Slit-lamp image shows corneal opacification (black arrow) and the site of the AGV implant insertion (black dashed arrow) (a). An AS-OCT image shows the aqueous humor flow inside the AGV tube (arrow) and the endplate of the AGV (dotted lines) (b). An area of high intensity in the tube lumen under the scleral patch (arrow) (c) seen in an optical coherence tomography image. The green arrows in insets indicate the direction of the AS-OCT scans. AGV, Ahmed glaucoma valve; AS-OCT, anterior-segment optical coherence tomography.

corneal cloudiness. The IOP remained within the normal range for 22 months with antiglaucoma eye drops and self-ocular massage. We confirmed the flow from the tube into the AGV and the encapsulation around the plate of the AGV using AS-OCT 8 months after removing the occluded tissue in the AGV tube (Fig. 2e).

The results showed that the fibrous materials could be Soemmering's ring or Zinn's zonule, which might occlude the AGV tube and block outflow from the tube into the AGV. This case represents a new cause of late-onset tube occlusion of the AGV using intraocular endoscopy.

\section{Discussion and Conclusion}

The GDD is effective for treating refractory glaucoma, and its use has increased in recent years. The most common GDDs are the AGV implant and the Baerveldt glaucoma implant (BGI) (Abbott Laboratories, Abbott Park, IL, USA). Both devices effectively decrease the IOP. The Baerveldt glaucoma implant achieved a lower IOP on fewer medications and had a lower failure rate and lower rate of de novo glaucoma surgery at 5 years than the AGV. However, the BGI was associated with a higher rate of failure from hypotony. The AGV has a higher risk of high IOP (AGV $42 \%$ vs. BGI $23 \%, p<0.001$ ) [11]. The rate of fibrous bleb encapsulation after AGV surgery is higher than that after BGI surgery $[6,11]$. These differences cause high IOP after AGV surgery, and fibrous encapsulation is the common cause of AGV failure.

In the current case, the first suspicious cause of high IOP was bleb encapsulation in the late postoperative period after AGV surgery, but bleb needling around the endplate of the AGV was ineffective. We then suspected tube occlusion but could not observe the tip of the tube 


\section{Case Reports in Ophthalmology}
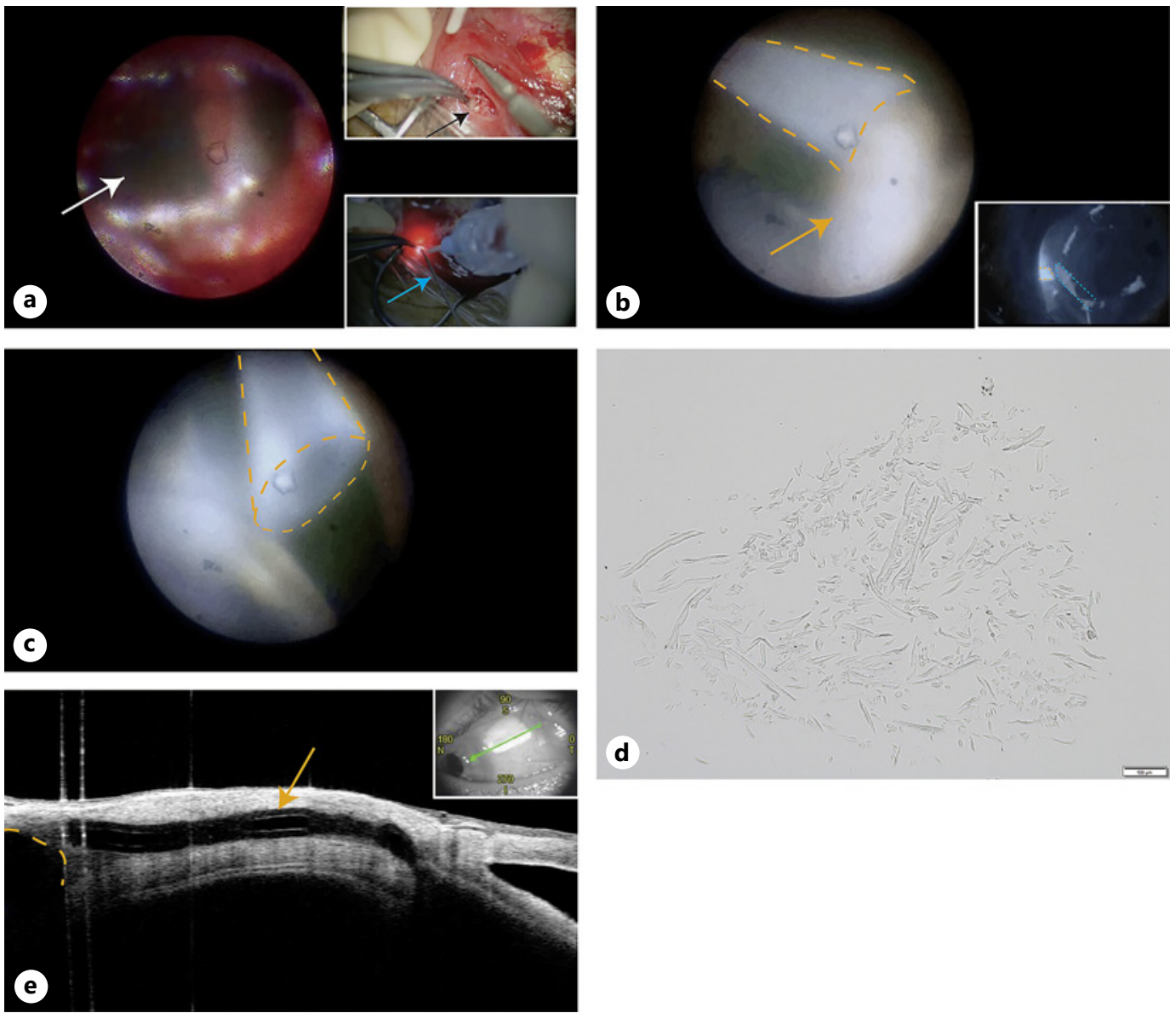

Fig. 2. Intraocular endoscopy image shows the AGV plate and the interior of the bleb. The white arrow shows the AGV plate (a). The surgical microscopy image shows the conjunctival incision created to insert the intraocular endoscope (top inset); the black arrow indicates the conjunctival incision. The blue arrow shows the intraocular endoscope (bottom inset). An intraocular endoscopy image shows the fibrous tissue occluding the tube. The orange dotted lines show the AGV tube. The arrow indicates the fibrous tissue (b). A surgical microscopic image (inset); the dotted lines indicate the intraocular endoscope. An intraocular endoscopy image shows the tube opening after the fibrous tissue is removed (c); the dotted lines indicate the AGV tube. Hematoxylin and eosin staining of the extracted fibrous tissues (d). An AS-OCT image 8 months after tube reconstruction. The arrow indicates the AGV tube (e). The orange dotted lines show the endplate of the AGV. The green arrow in inset indicates the direction of the AS-OCT scan. AGV, Ahmed glaucoma valve; AS-OCT, anterior-segment optical coherence tomography.

by slit-lamp microscopy because of severe corneal opacity. Previous studies have reported that occlusion of the AGV may occur early secondary to blood products in a retrograde fashion from bleeding in the sub-Tenon tunnel [7] or blood, posterior capsule, iris, or vitreous humor in an antegrade fashion [9]. In the current case, AS-OCT images showed aqueous humor flow inside the distal side of the tube. Although we found a high-intensity area in the tube lumen under the scleral patch, we could not detect the proximal tube occlusion around the tube tip through the ciliary sulcus. At this point, we did not know where the late occlusion of the AGV occurred.

AS-OCT is more widely used and useful for identifying glaucoma implant tube occlusion in the anterior chamber or outside of the eye as reported previously [12, 13]. However, 
AS-OCT cannot detect a high-quality posterior chamber tube because of the backscattering effect of the posterior iris pigment epithelium [14], especially in Asian eyes. Only 1 case reported that AS-OCT showed the obstruction of the tube posterior to the iris because iris atrophy reduced the backscatter of the infrared light [15]. The current patient had poor mydriasis and no iris atrophy, so we could not precisely detect the proximal tube occlusion around the tube tip through the ciliary sulcus.

Ultrasound biomicroscopy (UBM) provides higher quality images than AS-OCT of tubes positioned posterior to the iris. But UBM requires contact with the ocular surface and a trained examiner. The use of the water bath causes patient discomfort and a risk of infection or damage to the corneal graft. Our patient was susceptible to infection because he had just undergone a previous surgery and treatment. We could not perform UBM at that time.

However, while intraocular endoscopy might not be more widely available than AS-OCT or UBM, it can provide definitive proof, pathological results, and exact mechanisms of action. Intraocular endoscopy was useful for observing the proximal side of the tube in the AGV in a case such as this with an opaque cornea after keratoplasty and tube insertion into the ciliary sulcus. We identified a rare cause of late-onset occlusion after AGV surgery using intraocular endoscopy, that is, tube occlusion resulting from lens capsule-related tissue. Descemet stripping automated endothelial keratoplasty might affect the blockage of the materials without sufficient evidence. These findings made it possible to remove the tissue from the tube using lens capsule forceps, and the IOP has remained decreased for 1 year postoperatively. Intraocular endoscopy was helpful for resolving the tube occlusion of the $\mathrm{AGV}$, especially in a case with an opaque cornea after keratoplasty and tube insertion into ciliary sulcus.

\section{Acknowledgements}

We thank Lynda Charters of Medical International for English language editing. Lynda Charters agrees with her name appearing in this manuscript in the event that the manuscript is accepted for publication.

\section{Statement of Ethics}

The Ethics Committee of Osaka University Graduate School of Medicine approved this study protocol (approval reference No. 17330). The patient provided written informed consent to publish his case.

\section{Conflict of Interest Statement}

The authors have no conflicts of interest to declare in this report.

\section{Funding Sources}

The writing of the manuscript was supported in part by a Grant-in-Aid for Scientific Research C (No. 18K09406) (K.M.) and a Grant-in-Aid for Early-Career Scientists (No. 20K18379) (R.K.) from the Ministry of Education, Culture, Sports, Science, and Technology. 


\section{Author Contributions}

Conception or design of the work: K.M. Acquisition of data: R.K., K.M., M.K., and D.U. Analysis and interpretation of data for the work: R.K. and K.B. Drafting the work: R.K. and K.M. Revising it critically for important intellectual content: R.K., K.M., T.S., M.K., D.U., and M.N. Final approval of the version to be published: R.K., K.B., K.M., T.S., M.K., D.U., M.N., E.M., and K.N.

\section{Data Availability Statement}

All data generated or analyzed during this study are included in this article and its supplementary material files. Further inquiries can be directed to the corresponding author.

\section{References}

1 Gedde SJ, Herndon LW, Brandt JD, Budenz DL, Feuer WJ, Schiffman JC, et al. Postoperative complications in the Tube Versus Trabeculectomy (TVT) study during five years of follow-up. Am J Ophthalmol. 2012;153(5): 804-14.e1.

2 Steven JG, Joyce CS, William JF, Herndon LW, Brandt JD, Budenz DL, et al. Treatment outcomes in the Tube Versus Trabeculectomy (TVT) study after five years of follow-up. Am J Ophthalmol. 2012;153(5):789-803.

3 HaiBo T, Xin K, ShiHeng L, Lin L. Comparison of Ahmed glaucoma valve implantation and trabeculectomy for glaucoma: a systematic review and meta-analysis. PLoS One. 2015;10(2):e0118142.

4 Vinod K, Gedde SJ, Feuer WJ, Panarelli JF, Chang TC, Chen PP, et al. Practice preferences for glaucoma surgery: a survey of the American Glaucoma Society. J Glaucoma. 2017;26(8):687-93.

5 Rodriguez-Una I, Azuara-Blanco A, King AJ. Survey of glaucoma surgical preferences and post-operative care in the United Kingdom. Clin Exp Ophthalmol. 2017;45(3):232-40.

6 Budenz DL, Feuer WJ, Barton K, Schiffman J, Costa VP, Godfrey DG, et al. Postoperative complications in the Ahmed Baerveldt Comparison Study during five years of follow-up. Am J Ophthalmol. 2016;163:75-82.e3.

7 Coleman AL, Hill R, Wilson MR, Choplin N, Kotas-Neumann R, Tam M, et al. Initial clinical experience with the ahmed glaucoma valve implant. Am J Ophthalmol. 1995;120(1):23-31.

8 Feldman RM, El-Harazi SM, Villanueva G. Valve membrane adhesion as a cause of Ahmed glaucoma valve failure. J Glaucoma. 1997;6(1):10-2.

9 Hill RA, Pirouzian A, Liaw L. Pathophysiology of and prophylaxis against late Ahmed glaucoma valve occlusion. Am J Ophthalmol. 2000;129(5):608-12.

10 Matsushita K, Kawashima R, Nishida K. Development of an infrared monitor-guided bleb revision procedure. Case Rep Ophthalmol. 2020;11(2):234-41.

11 Christakis PG, Zhang D, Budenz DL, Barton K, Tsai JC, Ahmed IIK, et al. Five-year pooled data analysis of the Ahmed Baerveldt Comparison Study and the Ahmed Versus Baerveldt Study. Am J Ophthalmol. 2017;176: 118-26.

12 Kiddee W, Trope GE. Glaucoma tube imaging using anterior segment optical coherence tomography in patients with opaque cornea. J Glaucoma. 2013;22:773-5.

13 Nakakura S, Noguchi A, Noguchi S, Hirose Y, Niimi K, Tabuchi H, et al. Glaucoma implant tube lumen obstruction visualized using anterior segment optical coherence tomography. J Glaucoma. 2018;27:e64-7.

14 Radhakrishnan S, Rollins AM, Roth JE, Yazdanfar S, Westphal V, Bardenstein DS, et al. Real-time optical coherence tomography of the anterior segment at $1310 \mathrm{~nm}$. Arch Ophthalmol. 2001;119:1179-85.

15 Chua J, Mehta JS, Tan DT. Use of anterior segment optical coherence tomography to assess secondary glaucoma after penetrating keratoplasty. Cornea. 2009;28(2):243-5.

\section{Karger'}

